\title{
Association Between rRT-PCR Test Results Upon Admission and Outcome in Hospitalized Chest CT-Positive COVID-19 Patients: A Provincial Retrospective Cohort with Active Follow-up
}

\author{
Saeed Nemati ${ }^{1}$, Hamid Reza Najari ${ }^{2}$, Anita Eftekharzadeh (iD ${ }^{3}$, Amir Mohammad Kazemifar ${ }^{4}$, Ali \\ Qandian (iD ${ }^{5}$, Pedram Fattahi ${ }^{6}$, Sepideh Abdi ${ }^{1}$, Elnaz Saeedi ${ }^{1}$, Maedeh Zokaei Nikoo ${ }^{6}$ and Mohammad \\ Reza Rouhollahi (iD) 1 , ** \\ ${ }^{1}$ Cancer Research Center, Cancer Institute of Iran, Tehran University of Medical Sciences, Tehran, Iran \\ ${ }^{2}$ Department of Internal Medicine, School of Medicine, Qazvin University of Medical Sciences, Qazvin, Iran \\ ${ }^{3}$ Obesity Research Center, Research Institute for Endocrine Sciences, Shahid Beheshti University of Medical Sciences, Tehran, Iran \\ ${ }^{4}$ Treatment Affairs, Qazvin University of Medical Sciences, Qazvin, Iran \\ ${ }^{5}$ Communicable Disease Office, Health Affairs, Qazvin University of Medical Sciences, Qazvin, Iran \\ ${ }^{6}$ Student Research Committee, Qazvin University of Medical Sciences Qazvin, Iran \\ Clinical Cancer Research Center (CCRC), Milad Hospital, Tehran, Iran \\ Corresponding author: Cancer Research Center, Cancer Research Institute, Tehran University of Medical Sciences, Tehran, Iran. Email: mr-rouhollahi@razi.tums.ac.ir
}

Received 2020 December 04; Revised 2021 September 18; Accepted 2021 October 31.

\begin{abstract}
Background: The coronavirus disease 2019 (COVID-19) pandemic imposed the most devastating challenge on healthcare systems worldwide. Iran was among the first countries that had to confront serious shortages in reverse-transcriptase-polymerase chain reaction (RT-PCR) testing for severe acute respiratory syndrome Coronavirus 2 (SARS-CoV-2) and ventilators availabilities throughout the COVID-19 outbreak.

Objectives: This study aimed to investigate the clinical course of hospitalized COVID-19 patients with different real-time RT-PCR test results during the first three weeks of the outbreak in Qazvin province, Iran.

Methods: In this retrospective cohort study, patients with a positive chest computed tomography (CT) scan for COVID-19 who were admitted to all 12 hospitals across Qazvin province, Iran, between February 20 and March 11, 2020, were included and followed up until March 27, 2020. A multivariate logistic regression model was applied to compare the independent associates of death among COVID-19 patients. Then, patients were categorized into six groups based on admission to the intensive care unit (ICU) and rRT-PCR test status (positive, negative, or no test). Also, multilevel logistic regression was used to compare the odds of surviving in each group against the reference group (PCR negative patients not-received ICU) to show if the rational allocation of ICU occurred while its capacity is limited.

Results: In this study, we included 998 patients (57\% male; median age: 54 years) with positive chest CT scan changes. Among them, 558 patients were examined with rRT-PCR test and 73.8\% tested positive. Case fatality rate (CFR) was 20.68 and $7.53 \%$ among hospitalized patients with positive and negative tests, respectively. While only $5.2 \%$ of patients were admitted to the ICU, CFR outside ICU was 17.70 and $4.65 \%$ in patients with positive and negative results not admitted to the ICU, respectively.

Conclusions: Total CFR in all hospitalized COVID-19 patients in Qazvin province during the first three weeks of the pandemic was 11.7\%. Also, according to the results, the main risk factors included a positive rRT-PCR test, age more than 70 years, and having two or more comorbidities or just immunodeficiency disorders. Hence, the ICU admission criteria or prioritized ICU beds allocation should be considered with more emphasis on rRT-PCR results when the capacity of ICU beds is low.
\end{abstract}

Keywords: COVID-19, rRT-PCR, Pandemic, Cohort Study

\section{Background}

The first report of coronavirus disease 2019 (COVID-19) in Iran was officially announced on February 19, 2020, from the city of Qom in central Iran (1). Shortly thereafter, cases of infection with the novel coronavirus were reported from all over the country.

Although case fatality rate (CFR) in patients admitted to hospital and its associated clinical factors are some of the most substantial indicators that should be evaluated in COVID-19 pandemic, the complexity, and challenges, particularly in initial weeks of the pandemic, can 
cause lots of uncertainty. Among these complexities are: The rate of hospital and intensive care unit (ICU) admission (the former as the denominator of the CFR and the latter as one of the main courses of treatment), the criteria for hospital and ICU admissions, sensitivity and specificity of the diagnostic tests (including real-time reverse transcriptase-polymerase chain reaction (rRT-PCR) and computed tomography (CT scan), low access to and experience with diagnostic tests, and last but not least the treatment regimens administered. Furthermore, such factors as changing the definitions and criteria, inadequate hospital dataset standards, and incomplete data can dramatically change CFR assessment.

In the first wave of COVID-19 outbreak, when debates on the effect of anti-viral or any other drug regimen are still challenging, effective ICU services for the management of severe cases is crucial, although deficiencies may occur due to the number of cases or inappropriate allocation protocols. This situation provided a unique opportunity to not only assess the clinical pattern and outcome of the disease in Qazvin province, Iran but also, evaluate the performance of 12 hospitals throughout the province during the critical first three weeks of emergence the outbreak.

The results of such an assessment can further be employed as a basis for more precise hospital and ICU admission criteria aimed at saving more lives in the subsequent critical phases of the epidemic.

\section{Objectives}

Little data has been documented about the course and outcome of the affected patients in the country since the emergence of the COVID-19 epidemic in Iran. In this retrospective cohort study, we aimed to investigate the clinical pattern and outcome of patients with a primary diagnosis of COVID-19 but different rRT-PCR test results admitted to the hospitals and ICUs of Qazvin province Iran, from February 20, 2020, to March 11, 2020.

\section{Methods}

In this retrospective cohort study, patients with a primary diagnosis of COVID-19 (see definition) admitted to all 12 hospitals across Qazvin province, Iran, from February 20 , 2020 , to March 11, 2020, were included and followed up until March 27, 2020.

Upon the outbreak, the Qazvin University of Medical Sciences, as the provincial health governing body, provided an electronic data entry platform to collect epidemiologic and clinical data related to patients with COVID-19 and mandated all involved hospitals within the province to employ the database. Data were collected from patients' medical records and entered into the electronic database during the patients' admission period. This acquisition of data included epidemiological and clinical data, patients' exposure history, as well as the results of their COVID-19 rRT-PCR test and clinical outcome (i.e., discharge or death). After the discharge of patients, they were followed up by phone until March 27, 2020 to confirm the outcome.

This study was approved by the Research Ethics Committee of Qazvin University of Medical Sciences (Code: IR.QUMS.REC.1399.007). An informed consent was obtained from the patients upon admission.

\subsection{Inclusion Criteria}

Patients were admitted with a primary diagnosis of COVID-19 based on the INIGCDT (2). This edition, published on February 24, 2020, limited the indications for rRT-PCR testing to detect SARS-CoV-2 to those admitted with severe respiratory signs and symptoms or admitted patients with fever whose chest imaging revealed pulmonary infiltration (3). According to the guidelines of the INIGCDT, a primary diagnosis of COVID-19 is characterized by symptoms of fever, cough, or myalgia (referred herein as minor clinical criteria), coupled with either A) respiratory distress, low pulse oximetry reading ( $\mathrm{SpO} 2 \leq 93 \%$ ), respiratory rate (RR) of $>30$ (herein considered as major clinical criteria), or decreased level of consciousness; or B) patients being among high-risk groups who have an underlying medical condition along with suggestive chest X-ray or CT scan changes for COVID-19 (see below). Further laboratory and chest imaging studies were performed during the patients' admission course.

A definitive diagnosis of COVID-19 was made using rRTPCR to detect SARS-CoV-2 in patients' respiratory secretions. The test became available through the Pasteur Institute of Iran in early February 2020. Throat swab (nasopharyngeal and oropharyngeal) for initial diagnosis and lower respiratory secretion specimens (by induced sputum sample or in intubated patients) for definitive diagnosis were obtained from patients and sent for SARS-CoV-2 PCR test during their admission course. However, due to a serious shortage of these tests in Iran at the time, patients were not reexamined.

Fever was defined as an oral temperature of $>37.8^{\circ} \mathrm{C}$. Shortness of breath and decreased level of consciousness were defined upon the triage physician's clinical judgement. Underlying medical conditions that justified patients' admission, as proposed in the INIGCDT, included a history of cardiovascular disease, pulmonary disease, diabetes, hypertension, cancer, human immunodeficiency virus (HIV) infection, and organ transplantation. Chest Xray or CT scan changes suggestive of COVID-19 consisted of 
bilateral patchy infiltration with rapid progression toward ground glass opacity (GGO) $(2,3)$.

Patients were admitted to ICUs if they had persistent hypoxemia, decreased level of consciousness, hemodynamic instability, or hypercapnia.

Patients were discharged from the hospital if they had no fever for more than 48-72 hours, had an SpO2 of > 93\% while breathing ambient air, had improved clinical symptoms and signs, and showed remarkable improvement in their serial chest imaging.

For comparison, patients were categorized into four groups including: (1) survivors outside the ICU; (2) survivors inside the ICU; (3) non-survivors outside the ICU; and (4) non-survivors inside the ICU.

\subsection{Patients' Follow-up}

The patients who were admitted during the third week of the study period (5 - 7 March, 2020) were actively followed up using phone interviews on March 27. Among them, 436 patients (response rate $=76.7 \%$ ) were reached.

\subsection{Statistical Analysis}

Descriptive analyses were carried out using the median (IQR). Patients were categorized based on their week of admission, outcome inside and outside the ICU, and their rRTPCR testing status. The CFR was assessed for each group. The chi-square test was employed to assess differences in study variables among the different categories of patients.

A multiple logistic regression model was applied to identify the factors associated with death in COVID-19 patients. The odds ratio (OR) and 95\% confidence interval (CI) were determined for each contributing factor. Then, patients were categorized into six groups based on receiving the intensive care services and rRT-PCR test status (positive, negative, or no test). Also, multilevel logistic regression was used to compare the odds of surviving in each group against the reference group (PCR negative patients not-received ICU) to show if the rational allocation of ICU occurred while its capacity is limited. The constructed model was adjusted for sex, age, Charlson Comorbidity Index (CCI), calendar time, oxygen saturation, and type of hospital. The difference between compared groups was summarized in terms of OR. It also allowed us to calculate the mortality rate for each PCR/ICU group adjusted for factors associated with in-hospital mortality using the 'margins' and 'predict' commands in Stata software version 14.1.

Statistical analysis was performed using the Stata statistical software package (StataCorp. 2014. Stata Statistical Software: Release 14.1, College Station, TX: StataCorp LP). A P value $<0.05$ was considered as significant.

\section{Results}

The data of 1,197 patients admitted to hospitals in Qazvin province were entered into the related electronic database from February 20 to March 11, 2020. Among these patients, 191 patients who were PCR-negative and were admitted to non-COVID-19 wards were excluded from the analysis; however, their clinical course was followed up, as with all patients. The CFR among the latter group was 3.1\%, with none of them being admitted to ICUs. Furthermore, eight patients whose death occurred within the first 24 hours of their admission were excluded from the study (Figure 1).

A total of 998 patients ( $57 \%$ male) with a median age of 54 (IQR $25-75=25$ ) years were analyzed. Among them, 558 patients were tested for COVID-19, with 412 testing positive and 146 testing negative. ICU care was provided for $52 \%$ of patients, while the remaining $48 \%$ were isolated in the designated wards where they received low-flow oxygen and medical therapy (Table 1 ).

Comparing the study participants' characteristics between the four groups of survivors and non-survivors outside and inside the ICU, no sex difference in CFR was found $(\mathrm{P}=0.674)$. Among the non-survivors, except for those aged $<50$ years, death rates were higher in patients outside the ICU than inside; this difference was more prominent among patients aged $>70$ years (23.04 vs. $4.15 \%$, respectively). Notably, in the rRT-PCR test-positive group, a $20.68 \%$ CFR was observed (Table 2).

Comparing the study participants based on their PCR test results, it was found that while most tests had been carried out on individuals aged $>70$ years than the younger age groups, there was no statistically significant age difference between positive and negative rRT-PCR patients. Moreover, although patients receiving ICU services were tested more than non-ICU admitted patients, positive results were more prevalent in the latter group (Table 3 ).

The CFR among admitted patients in Qazvin province was $11.7 \%$ (Table 1); however, the CFR differed between the various groups (Table 4). A multiple logistic regression model including age group, sex, type of hospital(i.e., teaching or non-teaching; high volume or low volume), week of admission, ICU admission, co-existing disorders, and rRTPCR test results was used to determine the factors associated with death in patients. The results showed that age $>$ 70 years $(\mathrm{OR}=5.2 ; 95 \% \mathrm{CI}=2.9-9.1 ; \mathrm{P} \leq 0.001)$, immunodeficiency disorders $(\mathrm{OR}=4.3 ; 95 \% \mathrm{CI}=1.11-17.23 ; \mathrm{P}=0.035)$, ICU admission ( $\mathrm{OR}=11.5 ; 95 \% \mathrm{CI}=5.5$ - 23.9; $\mathrm{P} \leq 0.001)$, and having positive rRT-PCR test results $(\mathrm{OR}=5.8 ; 95 \% \mathrm{CI}$ $=2.7-12.5 ; \mathrm{P} \leq 0.001)$ were the main determinants of death in patients. Also, having two comorbidities or more was a weak risk factor for death $(\mathrm{OR}=1.8 ; 95 \% \mathrm{CI}=0.92$ - 3.06; 


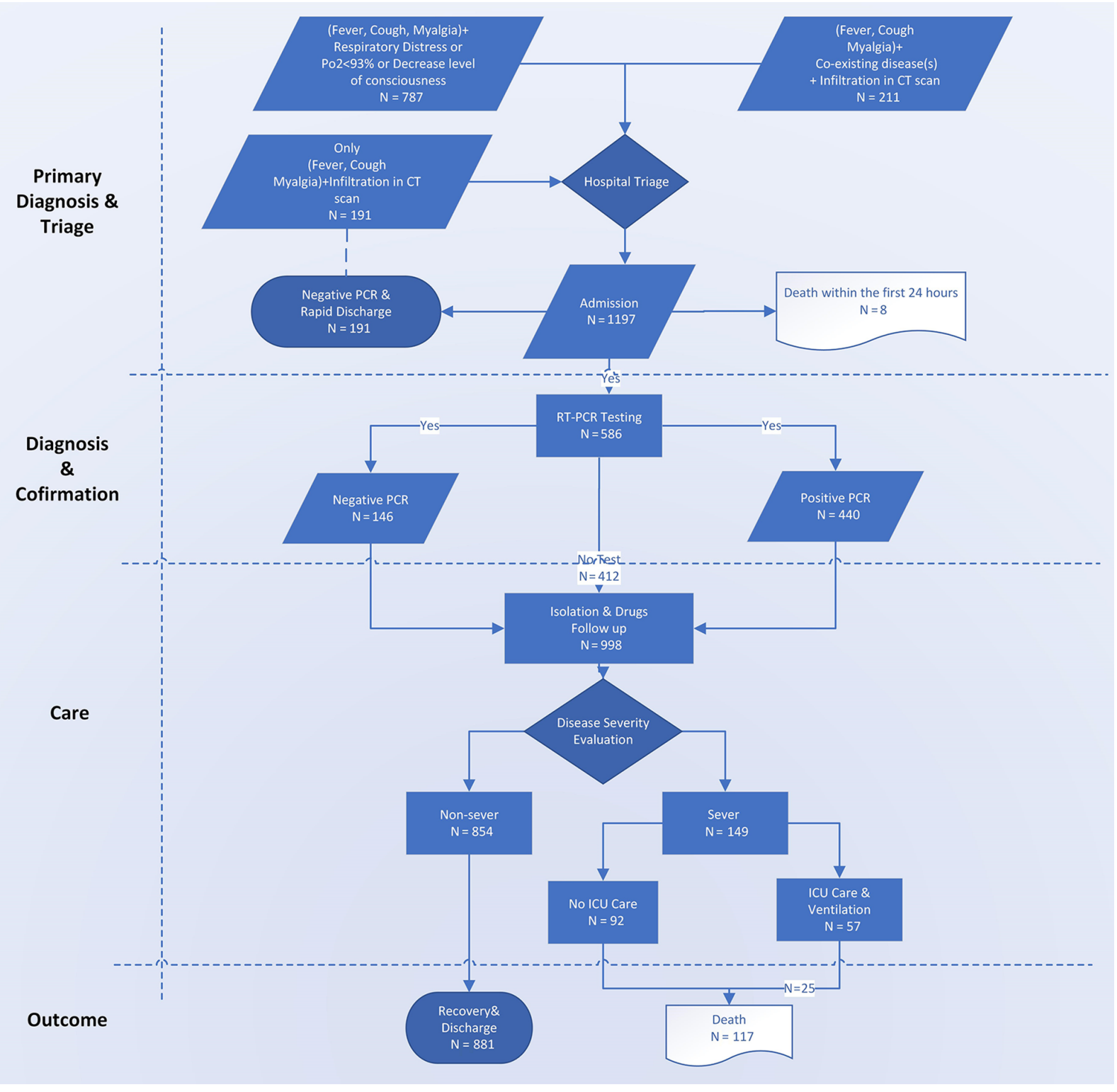

Figure 1. The patients' flow diagram

$\mathrm{P}=0.08$ ). After multi-level regression analysis in the six above-mentioned groups, only the two groups with positive rRT-PCR test results showed significantly higher mortality rates in comparison to the reference group after controlling the effects of confounding variables ( $O R=16.8 ; 95 \%$ $\mathrm{CI}=5.6-49.7 ; \mathrm{P}<0.001$ in $\mathrm{ICU}$ admitted group and $\mathrm{OR}=4.0$; $95 \% \mathrm{CI}=1.8-8.9 ; \mathrm{P}=0.001$ in the non-ICU admitted group).

\section{Discussion}

The present study was conducted in the first critical three weeks of the novel coronavirus outbreak in Qazvin province, Iran. The results showed that while CFR was 7.5\% among CT-diagnosed COVID-19 patients with typical respiratory symptoms but negative rRT-PCR results, this rate was as high as $20.7 \%$ in test-positive patients.

As shown in Table 1, a similar CFR was observed in both males and females. This finding is in line with the reports from Wuhan, China (3). On the other hand, the CFR in pa- 


\begin{tabular}{|c|c|c|c|c|}
\hline Variables & Week $1(N=116)$ & Week $2(\mathrm{~N}=399)$ & Week $3(N=483)$ & Total $(\mathrm{N}=998)$ \\
\hline \multicolumn{5}{|l|}{ Sex } \\
\hline Female & $57(49.14)$ & $168(42.11)$ & $204(42.24)$ & 429 (42.99) \\
\hline Male & $59(50.86)$ & $231(57.89)$ & $279(57.76)$ & $569(57.01)$ \\
\hline \multicolumn{5}{|l|}{ Age group $(y)$} \\
\hline$<50$ & $55(47.41)$ & $167(41.85)$ & $180(37.27)$ & $420(40.28)$ \\
\hline $51-60$ & $21(18.10)$ & $86(21.55)$ & $85(17.60)$ & $192(19.24)$ \\
\hline $61-70$ & $17(14.66)$ & $74(18.55)$ & $96(19.88)$ & $187(18.74)$ \\
\hline$\geq 70$ & $23(19.83)$ & $72(18.05)$ & $122(25.26)$ & $217(21.74)$ \\
\hline \multicolumn{5}{|l|}{ Admission criteria } \\
\hline Minor clinical criteria ${ }^{b}$ plus at least one major criteria ${ }^{c}$ & $92(79.31)$ & $317(79.45)$ & $378(78.26)$ & $787(78.86)$ \\
\hline Co-existing disorders OR immunodeficiency ${ }^{\mathrm{d}}$ & $24(20.69)$ & $82(20.55)$ & $105(21.74)$ & $211(21.14)$ \\
\hline \multicolumn{5}{|l|}{ rRT-PCR test status } \\
\hline Positive & $33(28.45)$ & $200(50.13)$ & $207(42.86)$ & $440(44.09)$ \\
\hline Negative & $20(17.24)$ & $49(12.28)$ & $77(15.94)$ & $146(14.63)$ \\
\hline Not tested & $63(54.31)$ & 150 (37.59) & $199(41.20)$ & $412(41.28)$ \\
\hline \multicolumn{5}{|l|}{ ICU admission } \\
\hline No & $108(93.10)$ & $377(94.49)$ & $461(95.45)$ & $946(94.70)$ \\
\hline Yes & $8(6.90)$ & $22(5.51)$ & $22(4.55)$ & $52(5.21)$ \\
\hline \multicolumn{5}{|l|}{ Co-existing disorders } \\
\hline Two or more comorbidities & $12(12.12)$ & $32(9.94)$ & $36(9.00)$ & $80(9.74)$ \\
\hline Cardiovascular diseases & $16(13.79)$ & $41(10.28)$ & $53(10.97)$ & $110(11.02)$ \\
\hline Diabetes & $13(11.21)$ & $43(10.78)$ & $49(10.14)$ & $105(10.52)$ \\
\hline Hypertension & $4(3.45)$ & $30(7.52)$ & $23(4.76)$ & $57(5.71)$ \\
\hline Chronic pulmonary diseases & $4(3.45)$ & $17(4.26)$ & $9(1.86)$ & $30(3.01)$ \\
\hline Immunodeficiency $^{\mathrm{d}}$ & $1(0.86)$ & $6(1.50)$ & $9(1.86)$ & $16(1.60)$ \\
\hline Chronic renal diseases & $1(0.86)$ & $4(1.00)$ & $5(1.04)$ & $10(1.00)$ \\
\hline Total & $30(25.86)$ & $111(27.82)$ & $124(25.67)$ & $265(26.55)$ \\
\hline \multicolumn{5}{|l|}{ Hospital type } \\
\hline Teaching & $92(79.31)$ & $317(79.45)$ & $360(74.53)$ & 769 (77.05) \\
\hline High volume non-teaching & $17(14.66)$ & $78(19.55)$ & $117(24.22)$ & $212(21.24)$ \\
\hline Low volume ${ }^{\mathrm{e}}$ non-teaching & $7(6.03)$ & $4(1.00)$ & $6(1.24)$ & $17(1.70)$ \\
\hline \multicolumn{5}{|l|}{ Outcome } \\
\hline Recovery & $104(89.66)$ & $352(88.22)$ & $425(87.99)$ & $881(88.28)$ \\
\hline Death & $12(10.34)$ & $47(11.78)$ & $58(12.01)$ & $117(11.72)$ \\
\hline
\end{tabular}

tients aged $>70$ years and in those who were ICU-admitted was higher compared to the reference group. Nevertheless, we did not find a significant increased death rate among COVID-19 patients with coexisting diseases. The exception, however, was in those with immunodeficiency disorders, in whom the CFR was about four times that of 
Nemati Set al.

\begin{tabular}{|c|c|c|c|c|c|c|c|}
\hline \multirow{2}{*}{ Variables } & & \multirow{2}{*}{ Total $(\mathrm{N}=\mathbf{9 9 8})$} & \multicolumn{2}{|c|}{ Non-survivors; $\mathrm{N}=117(11.7 \%)$} & \multicolumn{2}{|c|}{ Survivors; $\mathbf{N}=\mathbf{8 8 1}$ (88.3\%) } & \multirow{2}{*}{ PValue } \\
\hline & & & Outside ICU (N=92) & Inside ICU ( $N=25)$ & Outside ICU ( $N=854$ & Inside ICU (N=27) & \\
\hline \multicolumn{8}{|l|}{ Sex } \\
\hline & Female & $429(42.99)$ & $40(9.32)$ & $8(1.87)$ & $368(85.78)$ & $13(3.03)$ & \\
\hline & Male & $569(57.01)$ & $52(9.14)$ & $17(2.99)$ & $486(85.41)$ & $14(2.46)$ & 0.674 \\
\hline \multicolumn{8}{|c|}{ Age group $(y)$} \\
\hline & $51-60$ & $192(19.24)$ & $9(4.69)$ & $3(1.56)$ & $174(90.63)$ & $6(3.13)$ & \\
\hline & $61-70$ & $187(18.74)$ & $16(8.56)$ & $6(3.21)$ & $160(85.56)$ & $5(2.67)$ & \\
\hline & $\geq 70$ & $217(21.74)$ & $50(23.04)$ & $9(4.15)$ & $148(68.20)$ & $10(4.61)$ & $<0.001$ \\
\hline \multicolumn{8}{|c|}{ RT-PCR test status } \\
\hline & Positive & $440(44.09)$ & $74(16.82)$ & $17(3.86)$ & $344(78.18)$ & $5(1.14)$ & \\
\hline & Negative & $146(14.63)$ & $6(4.11)$ & $5(3.42)$ & $123(84.25)$ & $12(8.22)$ & \\
\hline & Not tested & $412(41.28)$ & $12(2.91)$ & $3(0.73)$ & $387(93.93)$ & $10(2.43)$ & $<0.001$ \\
\hline & Fever & $545(54.61)$ & $50(9.17)$ & $8(1.47)$ & $476(87.34)$ & $11(2.02)$ & 0.053 \\
\hline & Cough & $666(66.73)$ & $63(9.46)$ & $15(2.25)$ & $583(87.54)$ & $8(1.20)$ & $<0.001$ \\
\hline & Myalgia & $301(30.16)$ & $22(7.31)$ & $3(1.00)$ & $271(90.03)$ & $5(1.66)$ & 0.039 \\
\hline & Shortness of breath & $573(57.42)$ & $58(10.12)$ & $21(3.67)$ & $477(83.25)$ & $17(2.97)$ & 0.022 \\
\hline & $\mathrm{SpO} 2<93 \%$ & $612(61.32)$ & $61(9.97)$ & $17(2.78)$ & $513(83.82)$ & $21(3.43)$ & 0.166 \\
\hline & Unconsciousness & $31(3.11)$ & $4(12.90)$ & $7(22.58)$ & $12(38.71)$ & $9(29.03)$ & $<0.001$ \\
\hline & Minor clinical criteria & $185(18.54)$ & $13(7.03)$ & $3(1.62)$ & $166(89.73)$ & $3(1.62)$ & 0.343 \\
\hline & Major clinical criteria & $17(1.70)$ & $3(17.65)$ & $6(35.29)$ & $3(17.65)$ & $5(29.41)$ & $<0.001$ \\
\hline \multicolumn{8}{|c|}{ Co-existing disorders } \\
\hline & Cardiovascular disease & $110(11.02)$ & $20(18.18)$ & $6(5.46)$ & $81(73.64)$ & $3(2.73)$ & 0.001 \\
\hline & Diabetes & $105(10.52)$ & $15(14.29)$ & $4(3.81)$ & $84(80.00)$ & $2(1.91)$ & 0.189 \\
\hline & $\geq 2$ co-morbidities & $80(9.74)$ & $15(18.75)$ & $5(6.25)$ & $55(68.75)$ & $5(6.25)$ & $<0.001$ \\
\hline & Hypertension & $57(5.71)$ & $7(12.28)$ & $5(8.77)$ & $41(71.93)$ & $4(7.02)$ & 0.001 \\
\hline & Chronic pulmonary disease & $30(3.01)$ & $4(13.33)$ & $1(3.33)$ & $23(76.67)$ & $2(6.67)$ & 0.435 \\
\hline & Immunodeficiency & $16(1.60)$ & $2(12.50)$ & $3(18.75)$ & $10(62.50)$ & $1(6.25)$ & $<0.001$ \\
\hline & 1st & 116 & $8(6.90)$ & $4(3.45)$ & $100(86.21)$ & $4(3.45)$ & \\
\hline & 2nd & 399 & $36(9.02)$ & $11(2.76)$ & $341(85.46)$ & $11(2.76)$ & \\
\hline & 3rd & 483 & $48(9.94)$ & $10(2.07)$ & $413(85.51)$ & $12(2.49)$ & 0.903 \\
\hline \multicolumn{8}{|c|}{ Hospital type } \\
\hline & Teaching hospital & $769(77.05)$ & $71(9.23)$ & $22(2.86)$ & $658(85.57)$ & $18(2.34)$ & \\
\hline & High volume non-teaching & $212(21.24)$ & $20(9.43)$ & $2(0.94)$ & $184(86.79)$ & $6(2.83)$ & \\
\hline & Low volume non-teaching & $17(1.70)$ & $1(5.88)$ & $1(5.88)$ & $12(70.59)$ & $3(17.65)$ & 0.005 \\
\hline
\end{tabular}

the reference group (Table 3). In fact, the regression model showed that age affected death both independently and through a "corridor" of comorbidities. On the other hand, the adverse effects of major comorbidities were observed only in association with age, i.e., major comorbidities had no independent role in the CFR in this cohort of patients. In contrast, Guan et al. reported that, among hospitalized PCR-tested patients, the composite endpoint, including admission to ICU, invasive ventilation, and death, was higher in those who had known comorbidities (4). In addition, one meta-analysis previously reported that certain comorbidities were more prevalent in severe COVID-19 patients
(5).

This study is among the first investigations aiming to frame the current picture of the battle with the initial phase of the COVID-19 outbreak in one of Iran's provinces. The main strength of this study is its timing, i.e., reporting the clinical patterns of hospitalized patients during the early period of the outbreak, when the medical system is presumed not to be overloaded. Additionally, acquiring data from the electronic data entry platform, which was designated exclusively to collect data of COVID-19 patients during their course of admission, along with the large sample size add to the robustness of the study results. 


\begin{tabular}{|c|c|c|c|c|c|c|}
\hline \multirow{2}{*}{ Variables } & \multicolumn{3}{|c|}{ RT-PCR Test Status } & \multicolumn{3}{|c|}{ RT-PCR Test Results } \\
\hline & Tested $(\mathrm{N}=\mathbf{5 8 6})$ & Not Tested $(\mathrm{N}=412)$ & PValue & Test Positive $(\mathrm{N}=\mathbf{4 4 0})$ & Test Negative $(\mathrm{N}=146)$ & P Value \\
\hline \multicolumn{7}{|l|}{ Sex } \\
\hline Female & $243(56.64)$ & $186(43.36)$ & & $188(77.37)$ & $55(22.63)$ & \\
\hline Male & $343(60.28)$ & $226(39.72)$ & 0.248 & $252(73.47)$ & $91(26.53)$ & 0.283 \\
\hline \multicolumn{7}{|l|}{ Age group $(y)$} \\
\hline$<50$ & $221(54.98)$ & $181(45.02)$ & & $168(76.02)$ & $53(23.98)$ & \\
\hline $51-60$ & $101(52.60)$ & $91(47.40)$ & & $73(72.28)$ & $28(27.72)$ & \\
\hline $61-70$ & $119(63.64)$ & $68(36.36)$ & & $90(75.63)$ & $29(24.37)$ & \\
\hline$\geq 70$ & $145(66.82)$ & $72(33.18)$ & 0.005 & $109(75.17)$ & $36(24.83)$ & 0.908 \\
\hline \multicolumn{7}{|l|}{ ICU admission } \\
\hline Yes & $39(75.00)$ & $13(25.00)$ & & $22(56.41)$ & $17(43.59)$ & \\
\hline No & $547(57.82)$ & $399(42.18)$ & 0.014 & $418(76.42)$ & $129(23.58)$ & 0.005 \\
\hline \multicolumn{7}{|l|}{ Co-existing disorders } \\
\hline No & $405(55.25)$ & $328(44.75)$ & & $310(76.54)$ & $95(23.46)$ & \\
\hline Yes & $181(68.30)$ & $84(31.70)$ & $<0.001$ & $130(71.82)$ & $51(28.18)$ & 0.222 \\
\hline$\geq 2$ Comorbidities & $60(75.00)$ & $20(25.00)$ & 0.001 & $40(66.67)$ & $20(33.33)$ & 0.096 \\
\hline Cardiovascular diseases & $80(72.73)$ & $30(27.27)$ & 0.002 & $56(70.00)$ & $24(30.00)$ & 0.258 \\
\hline Chronic pulmonary diseases & $22(73.33)$ & $8(26.67)$ & 0.099 & $16(72.73)$ & $6(27.27)$ & 0.794 \\
\hline Diabetes & $74(70.48)$ & $31(29.52)$ & 0.010 & $54(72.97)$ & $20(27.03)$ & 0.653 \\
\hline Hypertension & $38(66.67)$ & $19(33.33)$ & 0.209 & $26(68.42)$ & $12(31.58)$ & 0.326 \\
\hline Immunodeficiency & $9(56.25)$ & $7(43.75)$ & 0.840 & $5(55.56)$ & $4(44.44)$ & 0.172 \\
\hline Chronic renal diseases & $7(70.00)$ & $3(30.00)$ & 0.466 & $5(71.43)$ & $2(28.57)$ & 0.822 \\
\hline \multicolumn{7}{|l|}{ Signs and symptoms } \\
\hline Any sign & $551(57.22)$ & $412(42.78)$ & $<0.001$ & $405(73.50)$ & $146(26.50)$ & $<0.001$ \\
\hline Fever & $293(53.76)$ & $252(46.24)$ & $<0.001$ & $224(76.45)$ & $69(23.55)$ & 0.445 \\
\hline Cough & $376(55.90)$ & $295(44.10)$ & 0.010 & $288(77.01)$ & $86(22.99)$ & 0.153 \\
\hline Myalgia & $159(52.82)$ & $142(47.18)$ & 0.013 & $119(74.84)$ & $40(25.16)$ & 0.934 \\
\hline Shortness of breath & $301(52.53)$ & $272(47.47)$ & $<0.001$ & $217(72.09)$ & $84(27.91)$ & 0.085 \\
\hline $\mathrm{SpO} 2<93 \%$ & $313(51.14)$ & $299(48.86)$ & $<0.001$ & $221(70.61)$ & $92(29.39)$ & 0.007 \\
\hline Unconsciousness & $22(68.75)$ & $10(31.25)$ & 0.241 & $11(50.00)$ & $11(50.00)$ & 0.006 \\
\hline \multicolumn{7}{|l|}{ Outcome } \\
\hline Recovery & $484(54.94)$ & $397(45.06)$ & & $349(72.11)$ & $135(27.89)$ & \\
\hline Death & $102(87.18)$ & $15(12.82)$ & $<0.001$ & $91(89.22)$ & $11(10.78)$ & $<0.001$ \\
\hline \multicolumn{7}{|l|}{ Admission week } \\
\hline $1 \mathrm{st}$ & $53(45.69)$ & $63(54.31)$ & & $33(62.26)$ & $20(37.74)$ & \\
\hline 2nd & $249(62.41)$ & $150(37.59)$ & & $200(80.32)$ & $49(19.68)$ & \\
\hline $3 r d$ & $284(50.80)$ & $199(41.20)$ & 0.006 & $207(72.89)$ & $77(27.11)$ & 0.011 \\
\hline
\end{tabular}

${ }^{a}$ Values are expressed as a proportion (\%) of the total number of patients for each week, as well as the total.

${ }^{\mathrm{b}}$ The chi-square test was used to compare groups.

c Immunodeficiency includes HIV infection and AIDS, cancer, immunodeficiency disorders, and receiving chemotherapy. 
Nemati Set al.

\begin{tabular}{|c|c|c|c|}
\hline Subgroups & Number of Deaths (\%) & Odds Ratio (95\% CI) & P Value \\
\hline \multicolumn{4}{|l|}{ Sex } \\
\hline Female & $48(11.19)$ & Reference & \\
\hline Male & $69(12.13)$ & $1.16(0.74,1.81)$ & 0.511 \\
\hline \multicolumn{4}{|l|}{$\operatorname{Age} \operatorname{group}(\mathrm{y})$} \\
\hline$<50$ & $24(5.97)$ & Reference & \\
\hline $50-59$ & $12(6.25)$ & $0.97(0.44,2.11)$ & 0.942 \\
\hline $60-69$ & $22(11.76)$ & $1.73(0.89,3.35)$ & 0.101 \\
\hline$\geq 70$ & $59(27.19)$ & $5.15(2.91,9.12)$ & $<0.001$ \\
\hline \multicolumn{4}{|l|}{ ICU admission } \\
\hline No & $92(9.73)$ & Reference & \\
\hline Yes & $25(48.08)$ & $11.46(5.53,23.86)$ & $<0.001$ \\
\hline \multicolumn{4}{|l|}{ RT-PCR test status } \\
\hline Negative & $11(7.53)$ & Reference & \\
\hline Positive & $91(20.68)$ & $5.75(2.65,12.48)$ & $<0.001$ \\
\hline Not tested & $15(3.64)$ & $0.74(0.30,1.84)$ & 0.531 \\
\hline \multicolumn{4}{|l|}{ Co-existing disorder } \\
\hline Without co-existing disorder & $67(9.14)$ & Reference & \\
\hline All & $50(18.87)$ & $1.4(0.91,2.34)$ & 0.110 \\
\hline$\geq 2$ Co-disorders & $20(25.02)$ & $1.82(0.92,3.60)$ & 0.081 \\
\hline Cardiovascular diseases & $26(23.64)$ & $1.57(0.86,2.87)$ & 0.186 \\
\hline Chronic pulmonary diseases & $5(16.67)$ & $1.28(0.43,3.79)$ & 0.438 \\
\hline Diabetes & $19(18.10)$ & $1.39(0.73,2.64)$ & 0.287 \\
\hline Chronic renal disease & $4(40.00)$ & $3.07(0.55,17.08)$ & 0.164 \\
\hline Immunodeficiency & $5(31.25)$ & $4.3(1.11,17.23)$ & 0.035 \\
\hline Hypertension & $12(21.05)$ & $0.99(0.44,2.20)$ & 0.991 \\
\hline \multicolumn{4}{|l|}{ Admission week } \\
\hline 1st & $12(10.34)$ & Reference & \\
\hline 2nd & $47(11.78)$ & $0.77(0.32,1.75)$ & 0.536 \\
\hline $3 \mathrm{rd}$ & $58(12.01)$ & $0.92(0.41,2.05)$ & 0.845 \\
\hline \multicolumn{4}{|l|}{ Type of hospital } \\
\hline Teaching hospitals & $93(12.09)$ & Reference & \\
\hline High-volume non-teaching & $22(10.38)$ & $0.61(0.32,1.11)$ & 0.140 \\
\hline Low-volume non-teaching & $2(11.76)$ & $0.23(0.03,1.55)$ & 0.113 \\
\hline
\end{tabular}

${ }^{\text {a }}$ A multivariate logistic regression analysis was performed to calculate the odds ratios ( $\left.95 \% \mathrm{CIs}\right)$.

${ }^{\mathrm{b}}$ Immunodeficiency includes HIV infection and AIDS, cancer, immunodeficiency disorders, and receiving chemotherapy.

${ }^{c}$ Low-volume hospitals indicate hospitals with less than 20 admitted COVID-19 patients.

However, the findings on the clinical patterns and outcomes of the patients need to be interpreted in the context of our limitations. Among these caveats, we must emphasize the low availability of PCR testing in Iran. In addition, we did not have access to data on the patients' laboratory results, their smoking status, or the medications received. The external validity of our results needs further consideration. Our cohort study was conducted in all public and private sector hospitals in Qazvin province during the first three weeks since the epidemic began. Thereby, the gener- 
alization of our results to other populations should be carried out with caution.

The applied diagnosis and treatment flowchart, as was first proposed by the INIGCDT (3), merits further explanation. These guidelines, which have been mainly adapted from the World Health Organization (WHO) guidelines (6, $7)$, proposed chest imaging as the first diagnostic step to screen patients who require prompt hospitalization amid shortages in RT-PCR test kits in Iran. As a result, all admitted patients with suggestive COVID-19 symptoms, with or without comorbidities, underwent chest imaging (specifically a chest CT scan) in Qazvin hospitals. Among them, $59.3 \%$ were tested with RT-PCR, of whom $24.9 \%$ had negative results. This proportion of negative test results is in line with previous findings. While there has been a significant correlation observed between throat swab and sputum sample viral loads (8), one study examining the bio-distribution of SARS-CoV-2 in different body tissues reported positive RT-PCR rates in only $72 \%$ of sputum specimens (9).

Examining the concordance between chest CT scan and PCR test results, a previous study from Wuhan, China reported that chest CT sensitivity was 97\% in RT-PCR-positive patients. On the other hand, in the PCR-negative patients, $75 \%$ had positive CT scan findings, $81 \%$ of whom were later considered as highly likely or probable cases of COVID-19 (10). Another study showed that the sensitivity of chest CT in diagnosing COVID-19 was significantly higher than RTPCR in their patients (98 vs. 71\%) (11).

When categorizing CT-diagnosed COVID-19 patients based on their RT-PCR test results, as expected, the worst scenario was reported in the group of patients with suggestive respiratory symptoms or underlying diseases who had positive PCR test results. These individuals accounted for $41 \%$ of ICU admissions throughout Qazvin province, with a 77\% CFR, even after receiving ICU services. On the other hand, the group with negative PCR test results who had suggestive clinical symptoms or underlying diseases accounted for 32\% of COVID-19-related ICU admissions in the province, among whom $71 \%$ recovered. It has been previously reported that negative PCR results may be due to lower viral load in patients' specimens $(12,13)$, which could provide a possible explanation for the lower CFR among the test-negative group. In the "no-test" group, which comprised CT-positive patients who were not tested for COVID-19 despite having suggestive clinical symptoms or coexisting diseases, 3.6\% were admitted to ICUs, of whom $66.7 \%$ eventually recovered. The overall CFR between notest group and patients with negative PCR results did not differ significantly. Based on the results of multilevel logistic regression analysis, in the first weeks of the epidemic, although PCR testing was more reserved for patients in a critical condition, its results should be presumed that a rather preemptive transfer of PCR positive patients to the ICU would partially explain the lower CFR in this group. Therefore, the criteria for ICU admission and prioritized allocation of the limited ICU beds should be identified (Table 5).

\begin{tabular}{|c|c|c|c|}
\hline Group & $\begin{array}{l}\text { In-Hospital } \\
\text { Mortality (95\% } \\
\text { CI) }\end{array}$ & $\begin{array}{l}\text { Odds Ratio (95\% } \\
\text { CI) }\end{array}$ & P-Value \\
\hline $\begin{array}{l}\text { Test negative/no } \\
\text { ICU }\end{array}$ & $6.1(5.7,6.9)$ & Reference & \\
\hline $\begin{array}{l}\text { Test positive/no } \\
\text { ICU }\end{array}$ & $19.0(18.2,19.8)$ & $4.0(1.8,8.9)$ & 0.001 \\
\hline No test/no ICU & $3.5(3.2,3.7)$ & $0.5(0.2,1.3)$ & 0.202 \\
\hline Test negative/ICU & $12.6(11.9,13.2)$ & $2.3(0.5,10.4)$ & 0.264 \\
\hline Test positive/ICU & $44.4(43.2,45.6)$ & $16.8(5.6,49.7)$ & $<0.001$ \\
\hline No test/ICU & $12.2(11.6,12.8)$ & $2.2(0.2,20.7)$ & 0.467 \\
\hline
\end{tabular}

Additionally, there was a group of patients with negative PCR results who also lacked major clinical symptoms or significant comorbidities. These patients had the lowest CFR, and none of them were ICU-admitted. These nonCOVID-19 hospitalized patients, who were not included in our analysis, may serve as a basis for comparing the admission course and outcomes of the study patients.

The importance of available ICU facilities in such an epidemic cannot be overemphasized. As officially announced by the Iranian Ministry of Health and Medical Education (MOHME) in 2018, there are a total of 8,264 ICU beds nationwide, and these were occupied by 453,891 patients during the year 2018 (occupancy rate: 55 patients/bed/year). In Qazvin province, which includes 1.6\% of Iran's population, there are 96 ICU beds available (7.7 beds/100,000 of the population), comprising $1.1 \%$ of the total ICU beds in Iran. In this study, among the 92 nonsurvivors treated outside ICUs, 63\% presented with shortness of breath, and $66 \%$ had low pulse oximetry readings. Among the 612 patients who presented an SpO2 of $<93 \%$, only $6.2 \%$ were admitted to ICUs. Considering that the ICU admission rate in this study was $5.2 \%$ (equal to 52 beds of total ICU beds in Qazvin province), it can be concluded that there is a need for at least 90 additional ICU beds throughout the province to save more lives. Interestingly, other studies have previously reported that while most COVID-19 patients present with a mild illness, about $14 \%$ progress to more severe forms of the disease and require hospitalization, with 5\% needing ICU care (14-16).

We also need to briefly mention the RT-PCR testing process. Due to the considerable shortage of PCR test kits in 
Iran, and according to the INIGCDT, physicians were encouraged to perform the test only in patients who were in a critical condition. Moreover, no re-testing was provided for patients with negative tests. While it has been reported that COVID-19 patients with more severe forms of the disease have higher viral loads and longer periods of viral shedding (6), the aforementioned testing protocol may have affected the results of the association between positive PCR test results and CFR.

In conclusion, we observed that COVID-19 patients hospitalized with mild symptoms, despite having positive chest CT changes and major comorbidities, were more likely to have negative rRT-PCR test results. Hence, there was a lower CFR and a more favorable outcome. Conversely, positive rRT-PCR test results were more prevalent in patients presenting with low $\mathrm{SpO} 2$ or unconsciousness, and they were strongly associated with increased odds of death among chest CT-positive patients. Considering the serious shortage in ICU capacity, preemptive transfer of more vulnerable rRT-PCR test-positive patients to the ICU might save their lives.

\section{Acknowledgments}

Authors wish to thank WE4H writing and editing, Vancouver, Canada, for providing medical writing and editing support in preparing this manuscript.

\section{Footnotes}

Authors' Contribution: S.N. drafted the manuscript, HR.N. conducted data acquisition, A.E., A.M., K.F. critically reviewed the manuscript, E.S. and S.A. performed statistical analysis, P.F. and M.Z. contributed in data collection and interpreting them and revising the manuscript, MR.R. contributed in developing the study concept and design and critically reviewed the manuscript.

Conflict of Interests: All authors declare that they have no conflict of interests.

Ethical Approval: This study was approved by the Research Ethics Committee of Qazvin University of Medical Sciences (Code: IR.QUMS.REC.1399.007).

Funding/Support: The current study was not received any fund or supports

Informed Consent: An informed consent was obtained from the patients upon admission.

\section{References}

1. Takian A, Raoofi A, Kazempour-Ardebili S. COVID-19 battle during the toughest sanctions against Iran. Lancet. 2020;395(10229):1035-
6. doi: 10.1016/S0140-6736(20)30668-1. [PubMed: 32199073]. [PubMed Central: PMC7138170].

2. Ives AR, Bozzuto C. Estimating and explaining the spread of COVID19 at the county level in the USA. Commun Biol. 2021;4(1):60. doi: 10.1038/s42003-020-01609-6. [PubMed: 33402722]. [PubMed Central: PMC7785728].

3. Azadmanesh $\mathrm{K}$, Jamaati $\mathrm{H}$, Javanian $\mathrm{M}$, Haj Abdulbaghi $\mathrm{M}$, Jan Babaie G, Hosseini S], et al. [Iran National Interim Guidance on COVID-19 Diagnosis and Treatment]. Tehran, Iran: Ministry of Health, Tratment and Medical Education; 2020. Persian.

4. Guan WJ, Liang WH, Zhao Y, Liang HR, Chen ZS, Li YM, et al. Comorbidity and its impact on 1590 patients with COVID-19 in China: a nationwide analysis. Eur Respir J. 2020;55(5):2000547. doi: 10.1183/13993003.00547-2020. [PubMed: 32217650]. [PubMed Central: PMC7098485].

5. Yang J, Zheng Y, Gou X, Pu K, Chen Z, Guo Q, et al. Prevalence of comorbidities and its effects in patients infected with SARS-CoV-2: A systematic review and meta-analysis. Int J Infect Dis. 2020;94:91-5. doi: 10.1016/j.ijid.2020.03.017. [PubMed: 32173574]. [PubMed Central: PMC7194638].

6. Liu Y, Yan LM, Wan L, Xiang TX, Le A, Liu JM, et al. Viral dynamics in mild and severe cases of COVID-19. Lancet Infect Dis. 2020;20(6):656-7. doi: 10.1016/S1473-3099(20)30232-2. [PubMed: 32199493]. [PubMed Central: PMC7158902].

7. World Health Organizatin. Laboratory testing for coronavirus disease (COVID-19) in suspected human cases: interim guidance. Geneva, Switzerland: World Health Organizatin; 2020.

8. Zhang Y, Dai C, Wang H, Gao Y, Li T, Fang Y, et al. Analysis and validation of a highly sensitive one-step nested quantitative realtime polymerase chain reaction assay for specific detection of severe acute respiratory syndrome coronavirus 2. Virol J. 2020;17(1):197. doi: 10.1186/s12985-020-01467-y. [PubMed: 33371898]. [PubMed Central: PMC7768088].

9. Wang W, Xu Y, Gao R, Lu R, Han K, Wu G, et al. Detection of SARS-CoV2 in Different Types of Clinical Specimens. JAMA. 2020;323(18):18434. doi: 10.1001/jama.2020.3786. [PubMed: 32159775]. [PubMed Central: PMC7066521].

10. Ai T, Yang Z, Hou H, Zhan C, Chen C, Lv W, et al. Correlation of Chest CT and RT-PCR Testing for Coronavirus Disease 2019 (COVID19) in China: A Report of 1014 Cases. Radiology. 2020;296(2):E32-40. doi: 10.1148/radiol.2020200642. [PubMed: 32101510]. [PubMed Central: PMC7233399].

11. Fang Y, Zhang H, Xie J, Lin M, Ying L, Pang P, et al. Sensitivity of Chest CT for COVID-19: Comparison to RT-PCR. Radiology. 2020;296(2):E1157. doi: 10.1148/radiol.2020200432. [PubMed: 32073353]. [PubMed Central: PMC7233365].

12. Xie X, Zhong Z, Zhao W, Zheng C, Wang F, Liu J. Chest CT for Typical Coronavirus Disease 2019 (COVID-19) Pneumonia: Relationship to Negative RT-PCR Testing. Radiology. 2020;296(2):E41-5. doi: 10.1148/radiol.2020200343. [PubMed: 32049601]. [PubMed Central: PMC7233363].

13. Liu J, Yu H, Zhang S. The indispensable role of chest $\mathrm{CT}$ in the detection of coronavirus disease 2019 (COVID-19). Eur J Nucl Med Mol Imaging. 2020;47(7):1638-9. doi: 10.1007/s00259-020-04795-x. [PubMed: 32246209]. [PubMed Central: PMC7118704].

14. World Health Organizatin. Clinical management of severe acute respiratory infection when novel coronavirus (2019-nCoV) infection is suspected: interim guidance. Geneva, Switzerland: World Health Organization; 2020.

15. Guan WJ, Ni ZY, Hu Y, Liang WH, Ou CQ, He JX, et al. Clinical Characteristics of Coronavirus Disease 2019 in China. $N$ Engl J Med. 2020;382(18):1708-20. doi: 10.1056/NEJMoa2002032. [PubMed: 32109013]. [PubMed Central: PMC7092819]. 
16. Allameh SF, Nemati S, Ghalehtaki R, Mohammadnejad E, Aghili SM, Khajavirad N, et al. Clinical Characteristics and Outcomes of 905 COVID-19 Patients Admitted to Imam Khomeini Hospital Complex in the Capital City of Tehran, Iran. Arch Iran Med. 2020;23(11):766-75. doi: 10.34172/aim.2020.102. [PubMed: 33220695]. 